\title{
Information tradeoffs in complex stimulus structure: Local and global levels in naturalistic scenes
}

\author{
MICHAEL VENTURINO \\ State University of New York, Geneseo, New York \\ and \\ DEBORAH A. GAGNON \\ State University of New York, Buffalo, New York
}

\begin{abstract}
An information tradeoff is an increased processing or utilization of information from one stim. ulus source at the expense of processing or utilization of information from a different source. An experiment was conducted to determine whether information tradeoffs occurred when subjects attended selectively to one of two different structural levels of naturalistic scenes. The subjects' attentional focus was directed to either the global or local structure of a scene (i.e., the scene or an object in the scene, respectively) either before or after presentation of a scene. They then had to use the information obtained from a $100-\mathrm{msec}$ exposure of the scene to choose between two forced-choice alternatives that described one of the levels. The nature of the alternatives was such that both alternatives adequately characterized one of the structural levels on the basis of physical and semantic relations within the scene. Results showed that the subjects were sig. nificantly slower and less accurate when their attentional focus and the forced-choice alternatives were at different levels of stimulus structure than when they were at the same level, providing evidence of an information tradeoff when different types of information from a scene were used. When processing information from a particular structural level, information from the other level either was less available or was not used efficiently. Furthermore, the information tradeoffs were more severe in the precue than in the postcue condition, indicating differences in the efficiency of the selectivity process. The results are interpreted with respect to the role of selective attention in processing complex stimuli such as naturalistic scenes.
\end{abstract}

The notion of selectivity in attention necessarily implies an information loss, in which some aspects of the stimulus environment are selected, processed, and transmitted at the expense of other aspects (Haber \& Hershenson, 1980 ; Kinchla, 1980). This selectivity is an inevitable consequence of the limits of the human information processing system and functions to allow extraction of a coherent, meaningful flow of information from the myriad of environmental signals and stimulation impinging on a perceiver (Broadbent, 1958; Kahneman, 1973). A notable effect of selectivity is an information tradeoff, in which

The first author was supported by funding from Project 7184 at the Human Engineering Division, Armstrong Aerospace Medical Research Laboratory, Wright-Patterson Air Force Base, Ohio. D.A.G. was supported by the Air Force Office of Scientific Research. We are very grateful to Irv Biederman for his comments, suggestions, encouragement, and for the generous use of his laboratory facilities. Thanks also to John Hilton for his programming efforts and assistance with the database, and to John Forester for his insightful comments. We would like to thank Lester Krueger, Ron Kinchla, Thomas Sanocki, and an anonymous reviewer for their thoughtful comments and suggestions on the mansucript. Correspondence concerning this article should be addressed to Michael Venturino, Department of Psychology, State University of New York at Geneseo, Geneseo, New York 14454-1401 (e-mail: venturin@geneseo.bitnet). increased processing or use of information from one stimulus source is associated with decreased processing or use of information from a different source, usually manifested through slower reaction times (RTs) and reduced accuracy (Kinchla, Solis-Macias, \& Hoffman, 1983; Posner, Nissen, \& Ogden, 1978; Sperling \& Melchner, 1978a, 1978b).

Kinchla et al. (1983) assessed the effects of selectivity on the use of information from different structural levels of a visual stimulus (i.e., local and global levels of a compound letter). By directing attention to one structural level and assessing usability of information from a different level, Kinchla et al. (1983) found that information from the emphasized level was utilized more rapidly and accurately than that from the other (nonemphasized) level. An interesting aspect of their experimental procedure was the use of a probability manipulation to direct subjects' attention to a specific structural level of the stimulus. This probability manipulation acted to establish a perceptual set effect, in which prior information about a stimulus facilitates perception or recognition (see Egeth, 1967, and Haber, 1966, for reviews). A set effect can be established by either specific features (Egeth \& Smith, 1967; Pachella, 1975), spatial location (Downing, 1988; Posner et al., 1978), or more general conceptual information (Carr \& 
Bacharach, 1976; Potter, 1975). The results of Kinchla et al. (1983) showed that the perceptual processing and organization of information can be facilitated by providing implicit information about a stimulus dimension such as a structural level (see also LaBerge \& Tweedy, 1964).

The purpose of the present experiment was to investigate information tradeoffs between levels of structure in naturalistic stimuli such as real-world scenes. Will the information tradeoffs that Kinchla et al. (1983) observed with reduced, artificial compound letter stimuli be obtained when the stimuli are naturalistic and complex? Establishing the existence of information tradeoffs between levels of structure in naturalistic scenes would demonstrate the generalizability of results from studies using simplistic artificial laboratory stimuli and show that the tradeoffs are not restricted to such stimuli. It would also aid in determining the role of selective attention in scene perception and processing, and validate the psychological reality of the levels themselves in naturalistic scenes. Finally, by temporally manipulating a cue, we were able to go one step further than previous research in examining the relationship between selectivity processes and stimulus structure. Specifically, we examined the efficiency of the selectivity process when the information for establishing a set was incompletely specified prior to stimulus presentation, compared with when the information was specified completely.

\section{Stimulus Organization: Local and Global Levels of Visual Form}

Forms and objects in the environment have an inherent structure that may be described at various levels of the part-whole hierarchy of organization. The local level of stimulus structure corresponds to the detailed features of a form, whereas the global level refers to the contours and gross features that contribute to the shape of an object or form.

The type of stimulus used in most experiments investigating stimulus structure has been compound letters or other such bilevel stimuli. A compound letter is a large letter composed of a configuration of smaller letters, such as the large $F$ formed from an appropriate configuration of small Hs shown in Figure 1a. The small letters constitute the local level of structure in such stimuli, whereas the large letter constitutes the global level. The levels of stimulus structure in these stimuli are orthogonal to, or uncorrelated with, one another. In other words, knowing the identity of elements at one structural level does not allow one to predict the identity of elements at the other level. This property of compound letters allows for easy manipulation of attention to one structural level and concomitant measurement of interference from the other level.

Most of the research investigating stimulus structure has been concerned with the question of whether global or local structure is processed first. Temporal precedence may not be dependent on stimulus structure, as Navon $(1977,1981)$ claimed, but may in fact depend on a num- (a)

$$
\begin{aligned}
& \text { HHHHHH } \\
& \mathrm{H} \\
& \mathrm{H} \\
& \mathrm{HHHHH} \\
& \mathrm{H} \\
& \mathrm{H} \\
& \mathrm{H}
\end{aligned}
$$

(b)

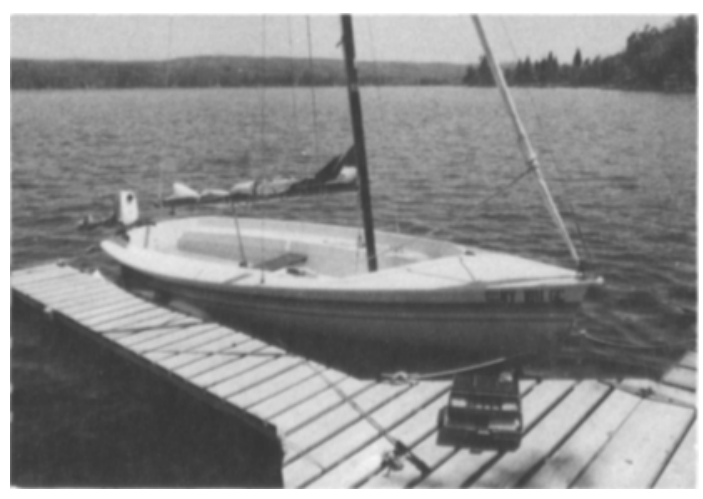

Figure 1. (a) An example of a compound letter typically used in studies of local and global stimulus structure; (b) a naturalistic scene typical of the stimuli used in the present experiment.

ber of factors related to visual aspects of the stimulus, such as image quality, relative conspicuity, discriminability, and visual angle of each of the levels (Hoffman, 1980; Kinchla \& Wolfe, 1979; Martin, 1979), the processing effort allocated to each level (Hoffman, 1980; Kinchla et al., 1983), and the structural level at which previous processing had occurred (Ward, 1982).

For the local-global distinction to be meaningful and useful, it must be shown to have psychological reality for complex naturalistic stimuli. It must be determined whether the attentional processes revealed by compound letters and other bilevel stimuli generalize to the complexity found in everyday scenes in our environment, and whether these structural levels play a role in scene perception and processing. In short, it would be useful to know whether naturalistic scenes have levels of structure that are functionally useful and amenable to experimental manipulation.

There are numerous and substantial differences between naturalistic scenes and artificial bilevel stimuli such as compound letters, which suggests that the generalizability of information tradeoff effects may not be a foregone conclusion. These differences are readily observed in comparing the compound letter and the scene in Figures $1 \mathrm{a}$ and $1 \mathrm{~b}$, respectively. Scenes are inherently more ambiguous with respect to their structure. For instance, the structural levels of naturalistic scenes are not orthogonal, and the boundary conditions for local and global levels may not be well defined, making the distinction between 
structural levels less clear. Naturalistic scenes also have a semantic component in which the referential meaning of many stimulus elements and their relationships play a role in organizing, perceiving, and interpreting the local elements (e.g., objects) and the scene itself (Biederman, 1981; Mandler \& Johnson, 1976). Relative to compound letters, the types of scenes and the physical properties of objects within a scene (e.g., saliency, size, color, number, etc.) vary widely. Unlike compound letters and bilevel stimuli that are usually drawn from a very limited domain of characters (allowing features to be primed and expected), naturalistic scenes are drawn from a relatively unlimited domain of possible stimuli for both local and global levels of structure. Naturalistic scenes are more complex by the sheer amount of information (number of objects, physical features, semantic features, etc.). Finally, we have considerably more experience with naturalistic scenes than with bilevel stimuli. The number and extent of these differences raise the obvious question of the generalizability of findings in which bilevel stimuli were used.

\section{Scene Perception}

Scenes are complex but coherent stimuli. A typical scene consists of texture, contours, forms, and objects, as well as physical and semantic relationships among these scene elements. Despite this complexity, sufficient information can be extracted from a brief presentation of a scene (e.g., 100-250 msec) to allow identification of the scene and objects within the scene (Biederman, 1981; Biederman, Rabinowitz, Glass, \& Stacy, 1974; Intraub, 1980; Loftus \& Bell, 1975). It has also been shown that semantic relations among elements in a scene are accessed as quickly as physical relations, suggesting that objects and their relationships are perceived together (Biederman, 1981). Implied in this ability is some underlying perceptual and cognitive organization; the notion of a schema (Bartlett, 1932) has been invoked as an explanation of one's ability to perceive scenes (Biederman, 1981; Biederman, Glass, \& Stacy, 1973; Mandler \& Johnson, 1976; Mandler \& Parker, 1976). Mandler and Johnson (1976) defined a schema as a cognitive structure, built up through experience, that organizes and gives meaning to incoming information. Biederman (1981) defined a schema for scene perception as representational knowledge that specifies both the items appropriate to a given scene and the physical and semantic relations among those items.

Efforts have been made to develop a taxonomy of the types of information in a scene. Biederman's approach (see Biederman, 1981) emphasizes the physical and semantic relationships that objects share within any particular scene. These relationships are specified in terms of physical constraints (e.g., physical laws and optical laws) and semantic constraints (e.g., probabilistic relations among objects within the context of a scene). ${ }^{1}$ Mandler's approach is a taxonomy of different types of information (Mandler \& Johnson, 1976; Mandler \& Parker, 1976). It includes the objects and their locations within a scene, the figurative detail of objects, and spatial composition information (areas of filled/empty space and the density of filled spaces). Both approaches maintain that naturalistic scenes consist of objects within some physical space whose lawful interrelationships constitute a meaningful pattern.

If the local level of stimulus structure consists of detailed information, then the local level of a naturalistic scene might arguably be the objects contained in it. ${ }^{2}$ If the global level of stimulus structure consists of organized patterns of objects, then the global level of a naturalistic scene might be the scene itself, characterized by the basic-level name of the scene (e.g., parking lot). Indeed, it is the lawful organization of relations among the objects within the scene that is essential in giving rise to the structure of a scene and provides the underlying structure of the scene itself. Note that local and global levels are defined only in relation to one another within a particular scene. A building may be a local element in a city block scene but may be the global element in a scene of a building.

The issue of concern to us is whether the information structure of naturalistic scenes allows organization into parts (local) and a whole (global) that can be attended to selectively. If so, then it is possible to determine how selectively processing a structural level in a scene affects perceiving and processing a scene.

\section{The Present Experiment}

The primary purpose of the experiment was to determine whether information tradeoffs in perceiving and processing scenes would occur when subjects attended selectively to one of two different structural levels of naturalistic scenes. In this way, we could determine whether the selectivity effects demonstrated by the use of compound letters (Kinchla et al., 1983) generalize to naturalistic scenes. Information tradeoffs would also provide evidence that stimulus structure is a meaningful construct for real-life scenes.

Another purpose of this experiment was to explore how varying the strength of the set effect might influence the selective process. This purpose was accomplished through the temporal manipulation of a cue specifying the location of local-level information; this manipulation completely or incompletely specified the information necessary for establishing perceptual set. In the precue condition, the location of local-level information was specified before presentation of the scene. In the postcue condition, the location of local-level information was specified after the scene was presented. In essence, the probability manipulation provided information regarding the relevance of features at a specific structural level, which served to establish a perceptual set. The cue manipulation provided information regarding the location of the object within the scene and was intended to modulate the effectiveness of the set effect by providing complete or incomplete information to use as a basis for selectivity. In the precue condition, since all of the necessary information for choosing between forced-choice alternatives was provided 
before exposure of the scene, the selectivity processes should be efficient, resulting in a severe information tradeoff. In the postcue condition, the selectivity processes should be less efficient because incomplete information was provided before scene exposure (i.e., no object location information was provided). Consequently, the information tradeoffs in the postcue condition should be less severe than in the precue condition.

The experimental task was a two-alternative forcedchoice task. Subjects determined which of two scene labels described a tachistoscopically presented scene or which of two object labels described a cued object within the scene. The subjects' attentional focus was directed to one of these two levels of scene structure by varying the proportion of trials in which the forced-choice alternatives described a particular structural level. This probability manipulation is similar to that used by Kinchla et al. (1983) to direct attention to different structural levels of compound letters. Success in directing attention to different levels would be manifested by information tradeoffs.

As mentioned above, there is a high degree of redundancy among the structural levels of a scene (Kinchla, 1977; Navon, 1981; Palmer, 1975). Because of this high correlation, or nonorthogonality, the information acquired from one level can potentially be used to infer or predict information at another level. For example, recognizing a stove allows one to predict a kitchen scene is present, and vice versa. (It was for this reason that Kinchla, 1977, developed orthogonal bilevel stimuli to investigate processing of stimulus structure.) To manipulate attention to structural levels effectively, it was necessary to remove or minimize the effects of the correlation between structural levels at the critical parts of the scene (i.e., the location of the cued object, or the entire scene itself). Therefore, we ensured that the information obtained at one structural level was probable in each of the forced-choice alternatives describing the other structural level. For example, the subjects could not use information obtained from the local level (e.g., a cued object such as a tackle box) to choose between alternatives at the global level (e.g., scene names such as a boat dock or a fishing pier) because the local-level information was as likely to occur in either of the global-level alternatives. Likewise, the subjects could not use the information at the global level (e.g., a dock scene) to choose between alternatives at the local level (e.g., a tackle box or a bucket), because both object alternatives were equally likely to appear in the scene. This procedural approach is analogous to that used by Reicher (1969) and Wheeler (1970) in studying the word-superiority effect.

We expected that the time to choose between alternative scene or object names would be fastest when the attentional focus and the forced-choice alternatives were at the same structural level. Substantial costs in time and errors were predicted when the attentional focus and forced-choice alternatives were at different structural levels (i.e., an information tradeoff). We also expected that the subjects' performance would be better in the precue than in the postcue condition. Although structural dominance and precedence in scene processing were of interest, no predictions regarding these issues were made because factors known to influence them were not controlled in the experiment.

Three factors were manipulated in the experiment. The first factor was a probability manipulation of the subjects' attentional focus to one of two different levels of stimulus structure: global or local (cf. Kinchla et al., 1983). In the global focus condition, the subjects chose between scene name alternatives on $80 \%$ of the trials within a block. In the local focus condition, the subjects chose between alternatives describing an object within a scene on $80 \%$ of the trials within a block. The second factor was a manipulation of the congruence between the structural level at which attention was focused and the level at which the subject was tested. The subjects' attentional focus and the forced-choice alternatives were either at the same stimulus structure level (same focus-test or match trials), or at a different structural level (different focus-test or mismatch trials). The third factor was a manipulation of the temporal nature of the local (object) cue, which occurred either before (precue) or after (postcue) the scene was presented. Attentional focus and focus-test congruence were within-subject manipulations, whereas temporal cue was a between-group manipulation.

\section{METHOD}

\section{Subjects}

Eighty subjects participated in the experiment. Of these, 71 were volunteers from the university community at the University of Minnesota at Minneapolis who were paid \$5 for participating in the 1-h experiment. The remaining 9 subjects were volunteers from the introductory psychology subject pool at the University of Minnesota who received class credit for participating. All subjects were native English speakers and had normal or corrected-to-normal vision.

\section{Stimuli and Apparatus}

The stimuli were 158 color slides of common, everyday scenes in the Buffalo, New York, area. Before being photographed, all scenes were carefully selected and composed so that there were no biases toward indoor or outdoor scenes, or toward private or public scenes. Additionally, all scenes were photographed within the constraints imposed by the forced-choice task, which dictated that each scene be describable by at least two labels, each of which could adequately characterize the scene on the basis of the component objects' physical and semantic relations within the scene. For example, a scene of a railroad yard could be described by either of the alternative word labels "freight yard" or "train depot."

An additional set of criteria pertained to the cued object within each scene: (1) the cued object could not violate any of the relational properties of either the actual scene presented or the scene characterized by the alternative label, (2) it could not be occluded or camouflaged in any way, (3) it had to be large enough to be readily noticeable within the scene, and (4) the cued object itself must be describable by at least two labels-its actual label and another object label that referred to an object that shared the acrual target object's physical and semantic relations in the scene. For example, in a scene of a boat dock in which a tackle box was the cued object, the alternative word labels were "tackle box" and "bucket." Both of the objects to which these labels refer share common rela- 
tional properties in the scene (e.g., size, location, and support) and are contextually probable within the scene structure itself.

The location of the scene on the screen was designated by four right-angle lines that corresponded to the four corner boundaries of the scene. The object location (local level) cue was a black dot (subtending $.42^{\circ}$ of visual angle) designating the location of the target object in the scene. The object location cue was temporally manipulated; the cue was presented by itself for the precue condition and was embedded in the mask for the postcue condition. The masking stimulus slide consisted of a random pattern of color patches.

The two alternative word labels for each trial were typed and made into slides. The labels were either two object names or two scene names. One label was placed on the left side and the other on the right side of each slide, which allowed for response compatibility with the two horizontally configured microswitches used by the subject in responding. Correct alternatives were balanced between left and right positions.

The experiment was conducted in a darkened room. Five Kodak Carousel projectors fitted with Gerbrands electronic tachistoscopic shutters were used, one each for the presentation of the frame, local level cue, scene, mask, and choice slides. An SWTP 6809 microcomputer controlled the stimulus presentation and response collection routines.

The slides were projected on a screen $8.5 \mathrm{ft}$ in front of the subject. The scenes subtended a visual angle of $10.78^{\circ} \times 7.40^{\circ}$ (width $x$ height). The mean visual angle of the cued object was $1.60^{\circ}$ $\times 1.68^{\circ}$ with a range of $.17^{\circ}-5.45^{\circ}$ wide and $.34^{\circ}-5.37^{\circ}$ high. The eccentricity of the cued object ranged from $.75^{\circ}$ to $9.38^{\circ}$ from the center of the scene, with a mean of $5.17^{\circ}$.

\section{Procedure}

The subjects participated in the experiment individually and were randomly assigned to either the precue or postcue group. They were allowed to dark adapt in the experimental room while the instructions were read. The instructions described the experimental procedure and emphasized the importance of speed and accuracy in the forced-choice task. In the global (scene) focus condition, the subjects were told that the alternative choices would be scene names in $80 \%$ of the trials and object names in $20 \%$ of the trials. These probabilities were reversed in the instructions for the local (object) focus condition. The subjects were encouraged to adopt a strategy that would enhance performance in each of these focus conditions. They were told to attend to the location of the object cue in the local focus condition. In the global focus condition, they were told to look in the center of the frame cue and get as much information about the scene as possible, and not to be distracted by the object cue.

The cue was intended to work in conjunction with the probability manipulation to differentially direct the subjects' attention to different aspects of the scene. In the local focus condition, the object location cue denoted a specific location in the scene, while the probability of choosing between object names $80 \%$ of the time induced the subjects to focus attention at that location. In the global focus condition, the probability of choosing between scene names $80 \%$ of the time induced subjects to distribute attention over a wide area framed by the four right angle lines denoting the scene boundaries. Note that the object location cue was present in the global focus trials. In those trials, however, the high probability of choosing between scene names should render it less effective in focusing attention narrowly at a specific location, since that would not be an efficient way of picking up information about the scene.

The subjects were given two blocks of 64 trials each, one in which attention was focused at the local level (local focus) and one in which it was focused at the global level (global focus). The focus order was counterbalanced across subjects, as was the forward and backward presentation order of slides. Two slides at the beginning and end of each block served as buffers and were not included in the data analysis. Eighty percent of the trials in each blocked focus condition ( 48 trials) contributed data to the focus-test match condition, in which the attentional focus and the forced-choice alternatives test were at the same structural level. The remaining $20 \%$ (12 trials) contributed data to the focus-test mismatch condition. Stimuli used in the mismatch trials were counterbalanced across subjects within each focus condition. Thus, across subjects, each stimulus was used in both the local and global focus conditions, and each slide appeared in both match and mismatch trials.

The subjects initiated a trial by pressing a key on the computer keyboard. One-half second after the keypress, the slide framing the scene location on the screen was presented for $500 \mathrm{msec}$. A scene slide was then presented for $100 \mathrm{msec}$ and was immediately succeeded by the color patch mask for 500 msec. The mask was followed by a slide containing the two alternative scene or object names for that trial. In the precue condition, the frame slide and the object location cue appeared simultaneously, before presentation of the scene. In the postcue condition, the object location cue followed the presentation of the scene stimulus and appeared simultaneously with the mask. The alternatives were displayed for $3 \mathrm{sec}$, during which time the subjects responded by pressing one of the two horizontally arranged microswitches with the index or middle finger of their preferred hand. RT was measured from the onset of the name alternatives to the microswitch press; both RT and accuracy were measured for each trial. Each subject was provided with feedback on the computer screen that included RT and an accuracy message.

The subjects received 15 practice trials before each block; these practice sets matched the relative proportion of scene and object choices present in the block of trials that followed. The practice allowed the subject to become familiar with the procedure as well as to adopt the level of focus to be used in the following block. None of the practice stimuli were used in the experimental blocks.

\section{RESULTS}

Analyses were performed on the mean RT and error data from the forced-choice task to determine whether an information tradeoff occurred as a function of selective attention. An information tradeoff was indicated by decreased performance when the forced-choice alternatives were at a structural level different from that of the subjects' attentional focus (focus-test mismatch trials) compared with when the alternatives and attentional focus were at the same structural level (focus-test match trials). The information tradeoff and temporal cue effect results are presented first, followed in turn by analyses of stimulus characteristics and attentional focus to stimulus structure.

\section{Information Tradeofis and Cue Effects}

The mean RT and error data are presented in Figures $2 a$ and $2 b$, respectively. The figures indicate that performance was worse when the subjects focused attention at one structural level but had to choose between forcedchoice alternatives describing the other structural level, compared with when the alternatives and attentional focus were at the same structural level. Also, the time required to choose between forced-choice alternatives was longer in the postcue than in the precue condition. The RT (correct responses only) and error data were analyzed by using a $2 \times 2 \times 2$ (cue $\times$ focus $\times$ focus-test congruence) mixed factors analysis of variance (ANOVA). 
(a)

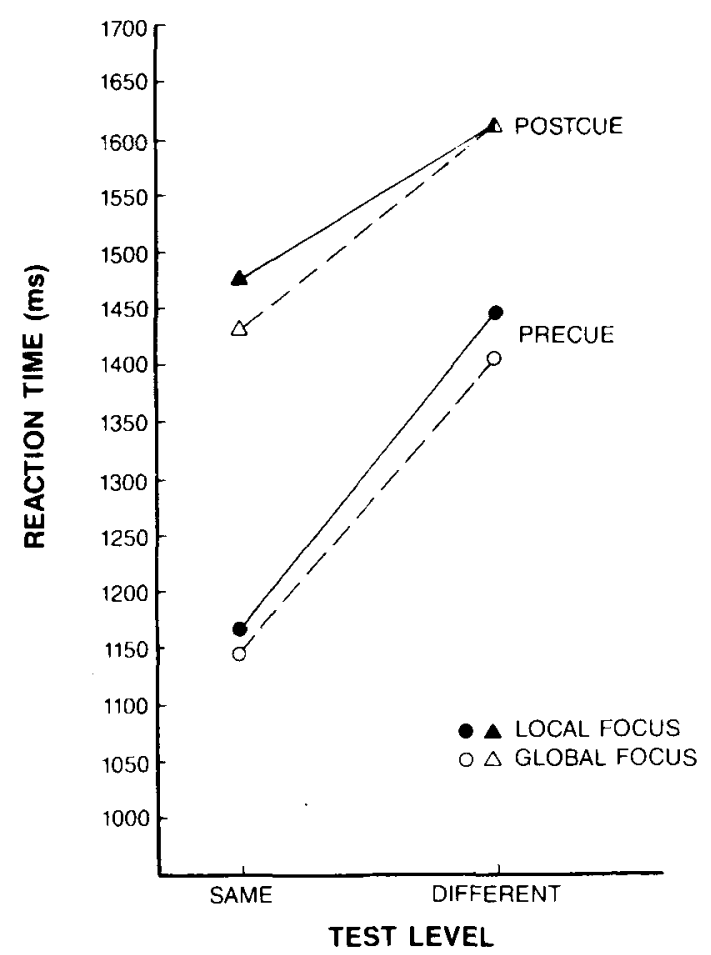

(b)

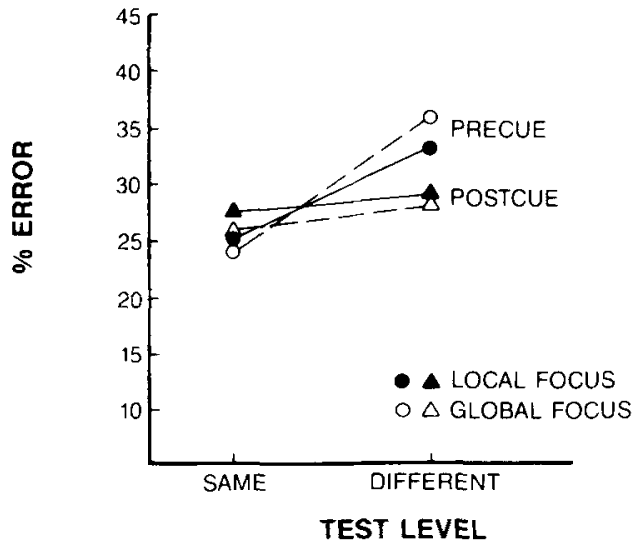

Figure 2. (a) Mean correct reaction time and (b) mean percent error, as a function of attentional focus, focus-test congruence, and cue. For the focus -test congruence factor, the forced-choice alternatives were either at the same or a different structural level from the subjects' attentional focus.

The subjects were significantly slower $[F(1,78)=$ $127.78, p<.0001]$ and less accurate $[F(1,78)=25.82$, $p<.0001]$ when their attentional focus and the forcedchoice alternatives were at different levels than when they were at the same level. This finding provides evidence for an information tradeoff in which there was a substantial cost associated with a focus-test mismatch (i.e., misdirected attention). Additionally, the subjects took significantly longer to choose between forced-choice alternatives in the postcue than in the precue condition $[F(1,78)=20.92$, $p<.0001]$.
Significant cue $\times$ focus-test congruence interactions for both $\operatorname{RT}[F(1,78)=5.19, p<.05]$ and errors $[F(1,78)=$ $12.92, p<.001]$ indicated that the information tradeoffs associated with misdirected attention were significantly greater in the precue condition than in the postcue condition. In the precue condition, attending to one structural level but having to respond on the basis of information from the other level required an additional $267 \mathrm{msec}$ and caused $10 \%$ more errors than attending and responding at the same structural level. In the postcue condition, the cost of misdirected attention was $166 \mathrm{msec}$ in RT and $2 \%$ in number of errors.

There were no other significant main effects or interactions $(p>.05)$. The lack of a significant focus $\times$ focus-test congruence interaction for either dependent variable indicated that the extent of information tradeoffs (i.e., the cost of misdirected attention) was equivalent whether the subjects focused attention at the scene level and had to use information from the object level or whether they focused at the object level and had to use information from the scene level.

\section{Stimulus and Control Variables}

Analyses were performed to determine whether stimulus characteristics (i.e., the distance of the cued object from the center of the scene and the size of the cued object), scene presentation order, or number of syllables in the forced-choice alternatives may have influenced performance.

Object eccentricity. The cued object in each scene occurred at varying locations within the scene boundaries. This analysis indicated how well subjects extracted and utilized information over a relatively large spatial area (the scene) when attention was focused at a specific spatial location within that area (the object). Only trials with scene labels as forced-choice alternatives were used in this analysis. Object eccentricity refers to the distance of the cued object from the center of the scene and was categorized into three levels: small $\left(0^{\circ}-3^{\circ}\right)$, medium $\left(3^{\circ}-6^{\circ}\right)$, and large $\left(6^{\circ}-10^{\circ}\right)$. To contrast the extreme distances, data from the small and large eccentricities were used in the analysis. A $2 \times 2 \times 2$ (cue $\times$ focus-test congruence $\times$ object eccentricity) ANOVA was performed on the data for both dependent variables. The RT and error data are presented in Table 1 . With respect to the RT data, there were two significant effects involving the eccentricity factor, a main effect of eccentricity $[F(1,78)=7.43, p<$ .01] and a significant cue $\times$ eccentricity interaction $[F(1,78)=4.46, p<.05]$. A breakdown of this interaction showed that in the postcue condition, accurately choosing between scene labels took $99 \mathrm{msec}$ longer when the cued object was at the edge of the scene than when it was in the center of the scene $[F(1,39)=14.64, p<$ $.001]$. In the precue condition, this difference was only $21 \mathrm{msec}(p>.05)$. The effect of object eccentricity on RT was equivalent whether the attentional focus and forced-choice alternatives were at the same or different levels $(p>.05)$. There was also a significant cue $\times$ focus-test congruence $x$ eccentricity interaction for er- 
Table 1

Mean RT (in Milliseconds) and Mean Percent Error for Object Eccentricity as a Function of Cue and Focus-Test Congruence

\begin{tabular}{|c|c|c|c|c|c|c|c|c|c|}
\hline \multirow[b]{4}{*}{ Focus-Test } & \multicolumn{4}{|c|}{ Precue } & \multicolumn{5}{|c|}{ Postcue } \\
\hline & \multicolumn{4}{|c|}{ Object Eccentricity } & \multicolumn{5}{|c|}{ Object Eccentricity } \\
\hline & \multicolumn{2}{|c|}{ Near } & \multicolumn{2}{|c|}{ Far } & \multicolumn{2}{|c|}{ Near } & \multicolumn{3}{|c|}{ Far } \\
\hline & RT & \% Error & $\mathrm{RT}$ & \% Error & RT & \% Error & RT & $\%$ & Error \\
\hline $\begin{array}{l}\text { Scene focus-Scene alternatives } \\
\text { Object focus-Scene alternatives }\end{array}$ & $\begin{array}{l}1,164 \\
1,438\end{array}$ & $\begin{array}{l}20 \\
26\end{array}$ & $\begin{array}{l}1,185 \\
1,478\end{array}$ & $\begin{array}{l}24 \\
35\end{array}$ & $\begin{array}{l}1,354 \\
1,589\end{array}$ & $\begin{array}{l}23 \\
29\end{array}$ & $\begin{array}{l}1,458 \\
1,656\end{array}$ & & $\begin{array}{l}27 \\
29\end{array}$ \\
\hline
\end{tabular}

Note-The data for eccentricity include only trials in which the forced-choice alternatives were scene names.

rors $[F(1,62)=7.02, p<.01]$. Analysis of this threeway interaction revealed a significant focus-test congruence $X$ eccentricity interaction for the precue condition $[F(1,31)=4.70, p<.05]$ but not for the postcue condition $(p>.05)$. In the precue condition, the subjects were less accurate in choosing between scene alternatives when attention was misdirected to a location near the edge of the scene ( $M=35 \%$ errors) than when attention was misdirected to a location near the center of the scene $[M=26 \%$ errors; $F(1,31)=6.07, p<.05]$. On the other hand, object eccentricity had little effect when the subjects directed attention to the scene level and had to choose between scene alternatives $(p>.05)$.

In summary, directing attention to a specific location at the edge of a scene had somewhat different effects on choosing between scene alternatives, depending on whether attention was directed before (precue) or after (postcue) the presentation of the scene. Directing attention to the edge rather than to the center of a scene caused the subjects to be slower in choosing between scene alternatives, but more so in the postcue than in the precue condition. Misdirecting attention to an object at the edge (compared with the center) of a scene caused reduced accuracy in choosing between scene alternatives in the precue condition but not in the postcue condition.

Object size. It must also be determined whether the size of the cued object affected the subjects' ability to use information from the object level in choosing between object label alternatives. Object size was categorized into two levels, based on object height and width: small (less than $2.5^{\circ}$ in either dimension) and large $\left(2.5^{\circ}-6^{\circ}\right.$ in either dimension). Only trials with object names as forced-choice alternatives were included in this analysis. A $2 \times 2 \times 2$ (cue $x$ focus-test congruence $x$ object size) ANOVA was per- formed on the RT and error data. Analysis of the RT data yielded a significant cue $\times$ focus-test congruence $\times$ size interaction $[F(1,67)=6.93, p<.01]$. These data are presented in Table 2 . This interaction was analyzed to determine the effects of the focus-test congruence $x$ size effects in each of the cue conditions. Object size had little effect on choosing between object alternatives in the precue condition, regardless of whether attention was directed to the scene or object level $(p>.05)$. In the postcue condition, there was a significant focus-test congruence $\times$ size interaction $[F(1,35)=6.00, p<.05]$. When attention was focused at the object level, the subjects were $98 \mathrm{msec}$ faster in choosing between object labels for a large object than for a small object $[F(1,39)=6.14$, $p<.05]$. When attention was focused at the scene level, the subjects were $30 \mathrm{msec}$ slower in choosing between object labels for a large object than for a small object $(p>$ $.05)$. There were no significant effects of object size on error rate $(p>.05)$.

Control variables. ANOVAs performed on both the RT and error data showed no systematic bias due to presentation order of the scenes. Analyses also showed that the number of syllables in the correct and alternative scene labels, and in the correct and alternative object labels, were equivalent across the levels of each of the three independent variables $(p>.05)$. Thus, the RT and error differences reported above are due to the experimental manipulations and not to differences in presentation order or syllable length.

\section{Stimulus Structure}

In order to assess whether any asymmetries in information tradeoff effects across levels of stimulus structure occurred in extracting and using information from natu-

Table 2

Mean RT (in Milliseconds) and Mean Percent Error for Object Size as a Function of Cue and Focus-Test Congruence

\begin{tabular}{|c|c|c|c|c|c|c|c|c|}
\hline \multirow[b]{4}{*}{ Focus-Test } & \multicolumn{4}{|c|}{ Precue } & \multicolumn{4}{|c|}{ Postcue } \\
\hline & \multicolumn{4}{|c|}{ Object Size } & \multicolumn{4}{|c|}{ Object Size } \\
\hline & \multicolumn{2}{|c|}{ Small } & \multicolumn{2}{|c|}{ Large } & \multicolumn{2}{|c|}{ Small } & \multicolumn{2}{|c|}{ Large } \\
\hline & RT & \% Error & RT & \% Error & RT & \% Error & RT & \% Error \\
\hline Object focus-Object alternatives & 1,179 & 25 & 1,149 & 24 & 1,499 & 28 & 1,401 & 25 \\
\hline Scene focus-Object alternatives & 1,439 & 36 & 1,296 & 39 & 1,610 & 29 & 1,640 & 25 \\
\hline
\end{tabular}

Note-The data for object size include only trials in which the forced-choice alternatives were object names. 
ralistic scenes, performance in the focus-test match conditions was compared. Thus, performance when the subjects focused on the scenes and chose between scene alternatives was compared with performance when the subjects focused on an object and chose between object alternatives. In the precue condition, choosing between scene alternatives took $1,147 \mathrm{msec}$ when attention was directed to the scene level; choosing between object alternatives took $1,171 \mathrm{msec}$ when attention was directed to the object level. The difference of $24 \mathrm{msec}$ was not significant $[F(1,39)<1]$. In the postcue condition, the subjects took $1,429 \mathrm{msec}$ to choose between scene alternatives when attention was directed to the scene level and $1,475 \mathrm{msec}$ to choose between object alternatives when attention was directed to the object level. The difference of $46 \mathrm{msec}$ was also not significant $[F(1,39)=1.22]$. The error rates for the focus-test match trials were equivalent in both the global and local focus conditions $(p>$ $.05)$ and for both the precue and postcue conditions ( $p>$ $.05)$. When attention and the forced-choice alternatives were at the same structural level, the subjects were as accurate in choosing between scene names as they were in choosing between object names, regardless of whether the cue appeared before or after presentation of the scene.

\section{DISCUSSION}

The present results indicate that the efficiency with which information from scenes can be used is a function of selective attention to the structure of the scene. When attention was directed to a particular structural level of a naturalistic scene, either local or global, information from that level was used relatively quickly and effectively in choosing between two likely alternatives describing that structural level. When attention was misdirected, information from the unemphasized level was either not available or not used efficiently, as evidenced by the fact that there were substantially more errors and longer RTs in choosing between alternatives describing the unemphasized level. The tradeoffs in selectively processing information from the different structural levels are portrayed in Figure 3. As can be seen in this figure, better performance in choosing between scene alternatives was associated with poorer performance in choosing between object alternatives, and vice versa. The only exception to this is the error rate for the postcue condition, in which no tradeoff was evident. This figure also shows that the information tradeoffs were more severe in the precue than in the postcue condition, which is indicated by the length of the tradeoff functions. Despite the very different task and types of stimuli used in this experiment, the tradeoff functions are remarkably similar to those obtained by Kinchla et al. (1983). The information tradeoffs associated with selective attention that had previously been observed with artificial, reduced stimuli were observed in the perception and use of information from complex, informationally rich naturalistic scenes. These findings demonstrate the generalizability of selectivity effects obtained (a)

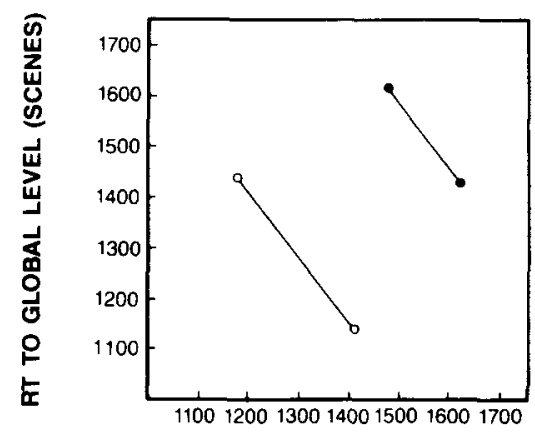

RT TO LOCAL LEVEL (OBJECTS)

(b)

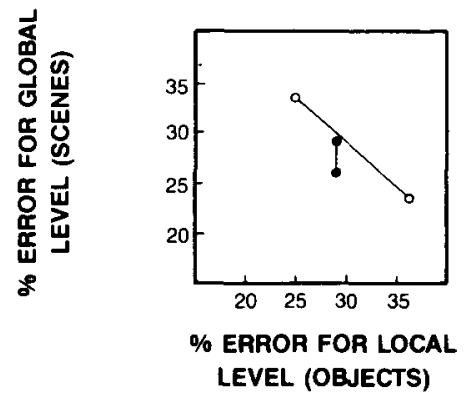

Figure 3. Information tradeofis for reaction time (RT) and percent error for the precue (open points) and postcue (closed points) conditions. The open and closed points in the upper left are from the local focus trials, and the points in the lower right are from the global focus trials.

by using abstract and artificial bilevel stimuli such as compound letters (Kinchla et al., 1983). Additionally, the results show that complex naturalistic (and "experimentally noisy") stimuli can be meaningfully organized into a hierarchical part-whole structure that can be manipulated in the laboratory.

\section{Information Tradeoffs}

The information tradeoffs may be interpreted in terms of a set effect that was engendered by the probability manipulation (i.e., the probability of choosing between alternatives descriptive of one structural level). The subjects biased their selectivity processes to organize and interpret information from the scene that was most useful in choosing between the high probability forced-choice alternatives. It should be noted that the type of features or specific information to be used in the forced-choice task was never specified explicitly. In fact, given the tremendous variability of features at both structural levels, it was impossible to specify such information. For each scene stimulus, the subjects had to determine for themselves which features would be useful for perceiving and identifying the scene or object. The utility of information at one structural level for responding on the forced-choice task led the subjects to selectively process that informa- 
tion at the expense of information at a different level. It is apparent that the perceptual processing and organization of information can be facilitated by providing implicit information about a stimulus dimension such as structural level (Kinchla et al., 1983) as well as conceptual information such as a name or category (Carr \& Bacharach, 1976; Potter, 1975), specific physical features of the relevant stimulus (Egeth \& Smith, 1967; Pachella, 1975), or spatial location (Downing, 1988; Posner et al., 1978).

The data from this experiment do not allow the precise specification of either the information-processing mechanisms involved in selectivity or their locus. However, several researchers have noted that the nature of the selective process may involve a number of perceptual and cognitive mechanisms or processes (Carr \& Bacharach, 1976; Kinchla, 1980; Kinchla et al., 1983; Pachella, 1975). Such processes may include the selective extraction and organization of information on the basis of spatial frequency channels (e.g., Breitmeyer \& Ganz, 1976), or the selective organization of features in forming a perceptual $/ \mathrm{mem}$ ory representation (Banks \& Prinzmetal, 1976; Haber 1966; Harris \& Haber, 1963; Pachella, 1975). Pachella (1975) eloquently argued that perception consists of two types of processes: an analytic process that extracts information from the stimulus or its visual representation, and a synthetic, constructive process that interprets the extracted information. Within the context of the present experimental procedure, the selective processes may have operated to organize certain aspects of the informationally rich scene stimulus, allowing an efficient categorization and identification of features necessary to arrive at the meaning of the scene or object. The subjects could then use that information (i.e., semantic information) to determine which of the alternatives best characterizes the meaning of the stimulus at that structural level. It is possible that the subjects generated a verbal label for the stimulus and used that verbal label, rather than the visual stimulus itself or its meaning, as a basis for comparison with the forced-choice alternatives. This would not be an optimal strategy, however, due to the fact that the alternatives were highly similar in characterizing the scene or object, which would cause confusion and result in a more difficult choice. It is more likely that the subjects used the meaning of the extracted information as a basis for choosing alternatives.

Given the very different nature of information at local and global levels of structure, the subjects may have adopted different strategies in selecting and processing the features from the scenes and their visual representation that were implicitly specified by the probability manipulation. In the local focus condition, the local-level features were implicitly emphasized, and the subjects may have used a strategy (e.g., attention distribution or selective organization of detailed features) that enhanced the processing and organization of information elements from a small area around the location of the object, either from the scene itself or its visual representation. Such a strategy may have precluded the efficient processing and organi- zation of features necessary to determine spatial and semantic relationships existing in other parts of the scene and its visual representation. In the global focus condition, global-level information was implicitly emphasized, and the subjects may have used a different strategy, one that enhanced the processing and organization of information over a relatively large area. Such a strategy may have allowed the processing of spatial and semantic relationships among spatially disparate elements in the scene and its representation, but at the expense of processing detailed information at any specific location (i.e., the cued object). Tradeoffs in processing information using different attention distribution strategies is a basic concept underlying gradient models of attention (Eriksen \& St. James, 1986; Eriksen \& Yeh, 1985). Alternatively, processing tradeoffs in an image or visual representation may be analogous to Kosslyn's (1975) results regarding the scaling of mental images. That is, the present results may be interpreted in terms of a tradeoff between the field-ofview and level of resolution in a visual image. Visualizing a large area such as a scene may make the details of the smaller elements less accessible. Conversely, focusing on a small area of a visual representation (such as an object) may put the more global elements (such as the overall scene) outside the useful field-of-view of the image.

\section{Cue Effects: Modulating the Extent of Tradeofis}

The time required to choose between alternatives was significantly faster in the precue condition than in the postcue condition. Specifying all the information (i.e., relevant structural level + the location of the local-level information) necessary to choose between alternatives before presentation of the scene may have allowed the selective processes to be more efficient in extracting, organizing, identifying, and using information compared with when only some of the information (i.e., only the relevant structural level) was specified before scene exposure. However, the information tradeoffs were more severe in the precue than in the postcue condition, indicating that the selectivity processes were more efficient or complete in the precue condition.

Within the context of the present experiment, a more efficient selective process would be indicated by a more severe information tradeoff. The more the selected information is analyzed and organized, the more it becomes available to consciousness while the nonselected information becomes less available (Carr \& Bacharach, 1976). One might conceptualize this as the selectivity processes developing a momentum in which the information from the emphasized level benefits from continuously more processing. On the other hand, a less efficient selective process would result when there is uncertainty generated by incomplete specification of relevant information before scene exposure. The uncertainty may lead subjects to process features from both structural levels, which by definition is less selective. Thus, the extent of the information tradeoffs may have been modulated by an uncertainty factor, which itself was caused by differences in the com- 
pleteness of information specified before presentation of the scene. This is supported by differences in the severity of the tradeoffs for the precue and postcue conditions.

In the precue condition, the subjects may have adopted a strategy to process the scene and its visual representation in accordance with the probability manipulation before the scene was exposed, allowing the selectivity mechanisms to operate more effectively in processing information from the emphasized structural level, but at the expense of information at the unemphasized level. However, the consequences of the selectivity process proved costly when the subjects' attention was misdirected before the scene appeared; in a sense, the subjects got caught processing the wrong information. The momentum of the selective process produced a severe tradeoff (both RT and error rate) in which the subjects processed and organized information from one structural level but were required to use information from the other level to choose between alternatives. That is, the subjects took advantage of the set engendered by the probability manipulation, biasing the organization and interpretation processes to identify features and their relationships and achieve meaning for the emphasized level. But when the alternatives unexpectedly described the other structural level, the subjects had to retrieve features from the fading visual representation of the scene and use what was left of that extracted information to choose the alternatives that best characterized the information at that level. Support for the notion that an attentional distribution process was at least partially responsible for the information tradeoffs comes from the precue condition in which the subjects' attention was misdirected to the local structural level and the object cue was located at the edge of the scene. A narrow attentional focus at one edge of the scene and its representation made it difficult to process and organize spatial and semantic relationships from other parts of the visual representation of the scene, as evidenced by the higher error rate. The less efficient processing may be due to the relationship between image field-of-view and resolution noted above. Alternatively, it may be due to image scanning. Kosslyn (1973; Kosslyn, Ball, \& Reiser, 1978) has demonstrated a linear relationship between distance and RT in scanning a mental image. Perhaps the time taken to scan large distances in the image was long enough so that critical elements of the unemphasized information in the visual representation faded, causing a loss of information and, consequently, a higher error rate.

In the postcue condition, the subjects could only nominally take advantage of the set afforded by the probability manipulation, since they had no information about the location of the cued object when the scene was presented. This resulted in a high degree of uncertainty about the location of information necessary for choosing between object alternatives when they appeared, whether object alternatives were more probable or not. In this condition, the best strategy would have been to distribute attention in such a manner as to select, organize, and process at least some information from both structural levels. The information tradeoff between structural levels in the postcue condition indicated that the subjects processed information from the emphasized structural level but had difficulty in using information from the other level. However, the tradeoff was not as severe as that obtained in the precue condition. The subjects may indeed have organized and processed some information from both structural levels; in other words, the selectivity processes were not as efficient as in the precue condition.

In addition to attentional distribution strategies, the relatively large visual angles subtended by the scene stimuli used here indicate that peripheral vision may also have been a limiting factor in acquiring information from disparate parts of the scene. The lower resolution information provided by peripheral vision may have contributed less effectively to a representation of some aspects of the scene. Perceiving naturalistic scenes is necessarily determined by both attentional distribution and retinal structure.

\section{Structural Equivalence in Scene Stimuli}

The structure of information in naturalistic scenes lends itself to a hierarchical organization consisting of parts and an integrated whole. When attention was directed to either structural level of a scene, there was efficient use of information from that level. Although responding on the basis of global information was faster than responding on the basis of local-level information, the difference was not statistically significant.

Other research involving memory for local and global information from naturalistic scenes found differential recognition accuracy to be a function of exposure duration (Loftus, Nelson, \& Kallman, 1983). With brief exposures (i.e., $250 \mathrm{msec}$ ), global aspects of the scene were remembered more accurately than were detailed aspects. Longer exposures resulted in higher recognition levels for detailed aspects of the pictures. Although such results suggest a temporal effect in perceiving structural information from scenes, Loftus and his colleagues did not require subjects to attend selectively to a particular structural level of the scene. It may be that in the absence of selective attention to a specified structural level (i.e., what to look for), global information may be salient and "more usable" than local-level information early in the processing of the scene. The present findings argue that when attention is directed to a structural level, either local or global, information from that level is used efficiently and effectively. Use of information from the nonemphasized level is not as efficient or effective.

Mandler and Johnson (1976) also found that some types of information in scenes were remembered better than others. These authors interpreted their results in terms of the selective nature of a schema. That is, the schema of a particular context or scene emphasized the acquisition of some information such as that concerning the spatial relationships among objects, at the expense of other information such as that concerning the proportion of filled 
and empty space in a scene. The selected information was perhaps processed more deeply and resulted in a stronger memory trace.

With regard to structural levels in scenes, two aspects of the information tradeoffs obtained in the present study are noteworthy. First, the information tradeoff between structural levels in naturalistic scenes occurred whether attention was directed to the global or local level. Second, the extent of this information tradeoff, with respect to RT and error rate, was equivalent for both structural levels; the cost of misdirected attention was similar regardless of whether it was misdirected to the global or local level. When attention was directed to a particular structural level, the strategies used in distributing attention allowed effective acquisition and use of that information. When attention was misdirected, these attention deployment strategies were deficient regardless of the structural level involved. Such results suggest that inefficient acquisition or use of information from a naturalistic scene is a property of selectivity rather than a function of stimulus structure or type of information in a scene. However, this interpretation must be viewed with caution because none of the factors known to influence structural precedence and dominance (e.g., discriminability, salience, etc.) were controlled in this experiment.

\section{Summary: Selective Attention and \\ Naturalistic Scenes}

The results of this experiment support the notion that the structure of naturally occurring scenes can be described at two levels, one level consisting of detailed objects and the other level consisting of global or holistic properties such as the scene itself. The ability to direct attention to one level of stimulus structure, demonstrated previously by using laboratory-developed compound letter stimuli (Hoffman, 1980; Kinchla et al., 1983; Ward, 1982), is also apparent with naturalistic scenes. How one distributes attention across a scene may determine which information from the scene is perceived and emphasized for further analysis. There appears to be a tradeoff associated with utilizing different types of information from a scene. When processing information from a particular structural level, information from the other level is less available or not used as efficiently. In essence, focusing attention on the forest makes it difficult to process the trees, and vice versa.

\section{REFERENCES}

BanKs, W. P., \& Prinzmetal, W. (1976). Configurational effects in visual information processing. Perception \& Psychophysics, 19, 361-367.

BARTLETT, F. C. (1932). Remembering: A study in experimental and social psychology. London: Cambridge University Press.

Biederman, I. (1981). On the semantics of a glance at a scene. In M. Kubovy \& J. R. Pomerantz (Eds.), Perceptual organization (pp. 213-253). Hillsdale, NJ: Erlbaum.

Biederman, I., Glass, A. L., \& Stacy, E. W. (1973). Searching for objects in real-world scenes. Joumal of Experimental Psychology, 97 , 22-27.
Biederman, I., Rabinowitz, J. C., Glass, A. L., de Stacy, E. W. (1974). On the information extracted from a glance at a scene. Journal of Experimental Psychology, 104, 597-600.

Biederman, I., Teitelbaum, R. C., Mezzanotte, R. J. (1983). Scene perception: A failure to find a benefit from prior expectancy or familiarity. Journal of Experimental Psychology: Human Perception \& Performance, 9, 411-429.

BREITMEYER, B. G., \& GANZ, L. (1976). Implications of sustained and transient channels for theories of visual pattern masking, saccadic suppression, and information processing. Psychological Review, 83, 1-36.

BroAdBent, D. E. (1958). Perception and communication. London: Oxford University Press.

CARR, T. H., Bacharach, V. R. (1976). Perceptual tuning and conscious attention: Systems of input regulation in visual information processing. Cognition, 4, 281-302.

DownING, C. J. (1988). Expectancy and visual-spatial attention: Effects on perceptual quality. Journal of Experimental Psychology: $\mathrm{Hu}$ man Perception \& Performance, 14, 188-202.

EgeTH, H. E. (1967). Selective attention. Psychological Bulletin, 67, 41-57.

Egeth, H. E., \& SMITH, E. E. (1967). Perceptual selectivity in a visual recognition task. Journal of Experimental Psychology, 74, 543-549.

ErIKSEN, C. W., \& ST. James, J. D. (1986). Visual attention within and around the field of focal attention: A zoom lens model. Perception \& Psychophysics, 40, 225-240.

ERIKSEN, C. W., \& YEH, Y. (1985). Allocation of attention in the visual field. Joumal of Experimental Psychology: Human Perception \& Performance, 11, 583-597.

HABER, R. N. (1966). The nature of the effect of set on perception. Psychological Review, 73, 335-350.

Haber, R. N., \& Hershenson, M. (1980). The psychology of visual perception (2nd ed.). New York: Holt, Rinehart \& Winston.

HARrIS, C. S., \& HABER, R. N. (1963). Selective attention and coding in visual perception. Joumal of Experimental Psychology, 65, 328-333.

HoFFMAN, J. E. (1980). Interaction between global and local levels of a form. Journal of Experimental Psychology: Human Perception \& Performance, 6, 222-234.

INTRAUB, H. (1980). Presentation rate and the representation of briefly glimpsed pictures in memory. Journal of Experimental Psychology: Human Learning \& Memory, 6, 1-12.

Kahneman, D. (1973). Attention and effort. New York: Prentice-Hall. KINCHLA, R. A. (1977). The role of structural redundancy in the perception of visual targets. Perception \& Psychophysics, 22, 19-30.

KINCHLA, R. A. (1980). The measurement of attention. In R. S. Nickerson (Ed.), Attention and performance VIII (pp. 213-238). Hillsdale, NJ: Erlbaum.

Kinchla, R. A., Solis-Macias, V., Hofman, J. E. (1983). Attending to different levels of structure in a visual image. Perception \& Psychophysics, 33, 1-10.

KINCHLA, R. A., \& WoLfE, J. M. (1979). The order of visual processing: "Top-down," "bottom-up," or "middle-out." Perception \& Psychophysics, 25, 225-231.

Kosslyn, S. M. (1973). Scanning visual images: Some structural implications. Perception \& Psychophysics, 14, 90-94.

KossLyN, S. M. (1975). Information representation in visual images. Cognitive Psychology, 7, 341-370.

Kosslyn, S. M., Ball, T. M., \& Reiser, B. J. (1978). Visual images preserve metric spatial information: Evidence from studies of image scanning. Journal of Experimental Psychology: Human Perception \& Performance, 4, 47-60.

LaBerge, D., \& Tweedy, J. R. (1964). Presentation probability and choice time. Journal of Experimental Psychology, 68, 477-481.

LofTUS, G. R., \& BeLL, S. M. (1975). Two types of information in picture memory. Joumal of Experimental Psychology: Human Learning \& Memory, 1, 103-113.

Loftus, G. R., NeLSON, W. W., \& Kallman, H. J. (1983). Differential acquisition rates for different types of information from pictures. Quarterly Journal of Experimental Psychology, 35A, 187-198.

MANDLER, J. M., \& JoHNSON, N. S. (1976). Some of the thousand words a picture is worth. Journal of Experimental Psychology: Human Perception \& Performance, 2, 529-540. 
Mandler, J. M., Parker, R. E. (1976). Memory for descriptive and spatial information in complex pictures. Journal of Experimental Psychology: Human Perception \& Performance, 2, 38-48.

MarTIN, M. (1979). Local and global processing: The role of sparsity. Memory \& Cognition, 7, 476-484.

NavoN, D. (1977). Forest before trees: The precedence of global features in visual perception. Cognitive Psychology, 9, 353-383.

Navon, D. (1981). The forest revisited: More on global precedence. Psychological Research, 43, 1-32.

PACHElla, R. G. (1975). The effect of set on the tachistoscopic recognition of pictures. In P. M. A. Rabbitt \& S. Dornic (Eds.), Attention and performance V (pp. 136-156). New York: Academic Press.

Palmer, S. E. (1975). Visual perception and world knowledge: Notes on a model of sensory-cognitive interaction. In D. A. Norman, D. E. Rumelhart, \& the LNR Research Group (Eds.), Explorations in cognition (pp. 279-301). San Francisco: W. H. Freeman.

Posner, M. I., Nissen, M. J., \& OGDen, W. C. (1978). Attended and unattended processing modes: The role of set for spatial location. In H. I. Pick \& E. Saltzman (Eds.), Modes of perceiving and processing information (pp. 137-157). Hillsdale, NJ: Erlbaum.

PotTer, M. C. (1975). Meaning in visual search. Science, 187, 965-966.

Reicher, G. M. (1969). Perceptual recognition as a function of meaningfulness of stimulus material. Joumal of Experimental Psychology, 81, 275-280.

Sperling, G., \& Melchner, M. J. (1978a). The attention operating characteristic: Examples from visual search. Science, 202, 315-318.

SPerling, G., \& Melchner, M. J. (1978b). Visual search, visual attention, and the attention operating characteristic. In J. Requin (Ed.), Attention and performance VII (pp. 675-686). Hillsdale, NJ: Erlbaum.
WARD, L. M. (1982). Determinants of attention to local and global features of visual forms. Journal of Experimental Psychology: Human Perception \& Performance, 8, 562-581.

WheEler, D. D. (1970). Processes in word recognition. Cognitive Psychology, 1, 59-85.

\section{NOTES}

1. With regard to the semantic constraints for any given scene, there is a limited class of objects that are of the appropriate size and that can occupy meaningful positions and locations to "fit" in with the naturally occurring context of the overall scene.

2. Some objects in a scene may contribute more than others to the physical layout of the scene. Although most objects in a scene will make some contribution to the semantic aspects of the scene, some objects may be more integral than others to interpreting the scene. In the present experiment, no violations of physical or semantic relationships were employed (cf. Biederman, 1981; Biederman, Teitelbaum, \& Mezzanotte, 1983). Objects occurred naturally within the scenes. Thus, the objects were actually more than objects-they were highly probable (but not necessary), correctly sized, correctly positioned objects in a scene. Examples of the local level might be a microphone in a scene depicting ham radio equipment, a lamp in a scene of a reading room, a paint can in a scene of a workbench, or a tackle box in a scene of a boat dock.

(Manuscript received April 23, 1991; revision accepted for publication April 7, 1992.) 